\title{
Clinico-Demographic Characteristics and Different Diagnostic Findings of Epilepsy Patients in a Specialized Hospital Outside Dhaka in Bangladesh
}

\author{
Abdus Salam¹, Md. Ruhul Quddus², Md. Sayedur Rahman Sheikh³, Md. \\ Arshad-ul-Azim ${ }^{4}$, Mohammad Enayet Hussain ${ }^{5}$ \\ ${ }^{1}$ Junior Consultant, Department of Neurology, Shaheed Sheikh Abu Naser Specialized Hospital, Khulna, Bangladesh; \\ ${ }^{2}$ Assistant Professor, Neurology Department, Shaheed Sheikh Abu Naser Specialized Hospital, Khulna, \\ Bangladesh; ${ }^{3}$ Registrar, Department of Neurology, Shaheed Sheikh Abu Naser Specialized Hospital, \\ Khulna, Bangladesh; ${ }^{4}$ Registrar, Department of Nephrology, Shaheed Sheikh Abu Naser Specialized \\ Hospital, Dhaka, Bangladesh; ${ }^{5}$ Assistant Professor, Department of Neurology, National \\ Institute of Neurosciences \& Hospital, Dhaka, Bangladesh
}

[Received: January 2015; Revised: March 2015; Accepted: June 2015; Published: January 2016]

\begin{abstract}
Background: Epilepsy is a common neurological conditions. It is a global problem affecting all ages, social classes, groups and countries. Objective: The purpose of the present study was to see the clinico-demographic characteristics and different diagnostic findings of epilepsy patients. Methodology: This cross sectional study was carried out in the Department of Neurology at Shaheed Sheikh Abu Naser Specialized Hospital, Khulna, Bangladesh from November, 2012 to February, 2014. The patients were recruited from the epilepsy clinic. Clinical diagnosis of epilepsy was made by meticulous history and relevant clinical examination by the investigator. Routinely electroencephalography (EEG) was advised and interpreted by the investigator and MRI was advised in appropriate cases. Results: A total number of 115 epilepsy patients were recruited of which $77(67.0 \%)$ were male and 38(33.0\%) were female. Most [95(83.7\%)] of the patients were young (age group 0 to 29 years) and had partial seizures $89(77.39 \%)$. Among these partial seizures most had complex partial seizure (CPS) [82(71.3\%)] of which $53(46.1 \%)$ had complex partial seizures-Temporal (CPS-T) and 29 (25.2\%) had complex partial seizures-extra-temporal (CPS-ET) origin. EEG could be done in 76 patients. Among them $37(48.7 \%)$ had different types of abnormalities. MRI could be done in 52 patients and were found to be abnormal in $32(61.5 \%)$ patients. Conclusion: This study revealed that localization related epilepsy (LRE) is more common but the etiology remains undetermined in many cases. Therefore, more effort and investigation facilities are needed to identify the etiology. The high number of extra temporal lobe epilepsy (Ex-TLE) needs further evaluation in bigger sample. [Journal of National Institute of Neurosciences Bangladesh, 2016;2(1): 3-9]
\end{abstract}

Keywords: Clinico-demographic characteristics; epilepsy; seizure

Correspondence: Dr. Abdus Salam, Junior Consultant, Department of Neurology, Shaheed Sheikh Abu Naser Specialized Hospital, Khulna, Bangladesh; Email: a.salamk51@gmail.com; Cell no.: +8801553567977

Conflict of interest: There is no conflict of interest relevant to this paper to disclose.

Funding agency: This research project was not funded by any group or any institution.

Contribution to authors: AS, MRQ and MSRS were involved in protocol preparation, data collection and literature search up to manuscript writing. AS, MAA and MEH were involved in preparation and revision of this manuscript.

How to cite this article: Salam A, Quddus MR, Sheikh MSR, Arshad-ul-Azim M., Hussain ME. Clinico-Demographic Characteristics of Epilepsy Patients in a Referral Level Hospital in Outside Dhaka City in Bangladesh. J Natl Inst Neurosci Bangladesh, 2016;2(1): 3-9

Copyright: (C2016 Salam et al. Published by Journal of National Institute of Neurosciences Bangladesh. This article is published under the Creative Commons CC BY-NC License (https://creativecommons.org/licenses/by-nc/4.0/). This license permits use, distribution and reproduction in any medium, provided the original work is properly cited, and is not used for commercial purposes.

\section{Introduction}

Epilepsy is a common neurological condition affecting people of all ages, race and social class. There are an estimated 50 million people with epilepsy in the world, 
of whom up to $75.0 \%$ live in resource-poor countries with little or no access to medical services or treatment ${ }^{1,2}$. The clinical scenario is almost similar in developing and developed countries, but the extent to which patients with epilepsy are recognized, investigated, and managed is different. Epidemiology, etiology, socio-cultural, and economic factors all contribute to these differences ${ }^{3}$.

Diagnostic accuracy is a particular problem in epilepsy as seizures are a symptom of diverse underlying cerebral etiologies and normally do not have any physical manifestations. Consequently a definitive diagnosis of epilepsy is often only be made after an extended period of follow up, as evidenced in the Rochester study and the National General Practice Study of Epilepsy (NGPSE), a community-based study of epilepsy in the United Kingdom. Moreover it has been found that 20 to $30 \%$ of those attending tertiary referral centers with refractory epilepsy do not in fact have epilepsy, with the most common differential diagnoses being pseudo seizures and syncope. As expected, neurologists are better at the diagnosis of epilepsy than non-specialists (mistake rate $5.6 \%$ vs. $18.9 \%$ ), but a misdiagnosis rate of $5 \%$ should be considered as the absolute minimum ${ }^{4}$.

Many people with epilepsy may not come to medical attention, due to ignorance or lack of awareness of the symptoms. This is particularly true for absence and minor complex partial seizures, which may only be recognized in retrospect following presentation with a generalized seizure. Indeed in one study of general practices only $20.0 \%$ of patients with seizures were suspected to have the diagnosis prior to medical consultation ${ }^{4}$. However, there is very little information on the types of epilepsy or on their clinical presentations, EEG records, or clinical outcomes. These are important for planning management and for developing wider services within the country.

Bangladesh is one of the densely populated countries in the world where infectious diseases, malnutrition and many chronic neurological disorders are quite common. Although there is no national statistics yet in the country but there are some hospital based studies that reflect to some extent the situation of epilepsy in Bangladesh. Studies in developed countries shows prevalence rate of about 5 per 1,000 populations whereas in developing countries it is higher. Men are more often affected than female and rural populations are affected more than the urban populations $s^{6}$. Based on the prevalence rate of 10 per 1,000 populations it is estimated that out of 150 million, there are at least 1.5-2.0 million people with epilepsy in Bangladesh?
The etiology varies with age. Birth trauma, birth asphyxia, central nervous system infections are common in neonate and infancy whereas head trauma, brain tumor, stroke, infections are common causes in middle aged and elderly ${ }^{1}$. Therefore the aim of this present study was to obtain a baseline profile of epilepsies, to determine the types of epilepsies and epileptic syndromes in patients attending the outdoor epilepsy clinic in a tertiary care hospital.

\section{Methodology}

This was a cross sectional study carried out in weekly epilepsy clinic of Department of Neurology at Shaheed Sheikh Abu Naser Specialized Hospital, Khulna from November 2012 to February, 2014 for a period of one year and three months. The epilepsy clinic provides comprehensive clinical care to epilepsy patients and is run by the investigator who obtained his training on epilepsy through Asian Epilepsy Academy (ASEPA) fellowship program. This is the only center in this division comprising 10 districts and 15,563,000 inhabitants ${ }^{8}$ which provides such type of facilities. Therefore patients are referred hear from all over the division. During this period patients with history of seizure disorder attended this clinic. All patients who had been seen consecutively in the epilepsy clinic were enrolled into the study. A preformed questionnaire was used containing information on age, sex, habitat, clinical history from patients and observers, examination findings, previous and current medications, result of EEG and imaging studies for data collection. A review of baseline clinical information, EEG reports, other investigations, and follow-up records was performed. If the information was insufficient, a further follow-up review was undertaken by recalling the patients and the care-givers by telephone calls. An EEG was advised routinely to all patients. EEG was performed at either first presentation or at any stage during the follow-up period. The EEG was obtained with a 32-channel digital machine with the electrodes placed in accordance with the international 10-20 system. In most cases recordings were obtained in both the awake and sleep states for 30 to 40 minutes. Photic stimulation and hyperventilation were done routinely. The EEGs were interpreted and reported by the investigator. The findings were grouped into two main categories 'normal' and 'abnormal'. The abnormal EEG was defined as the presence of interictal or ictal epileptiform discharges and/or the presence of abnormal background activity with focal or generalized, slow waves or excessive fast waves, 
abnormal for the age and state of the patients. EEG abnormalities were considered focal if there was a localized spike and sharp wave discharge or persistent focal slowing. Other investigations such as neuroimaging (MRI, CT-scan) were advised for all patients who were clinically and electrographically diagnosed as LRE, patients with symptomatic generalized epilepsy, late onset epilepsy and also for patients with clustered seizures, frequent seizures, isolated seizures, intractable epilepsy, and patients who had unexplained break-through seizures in otherwise well-controlled epilepsies. The available films were interpreted jointly by the radiologist and the investigator. All clearly abnormal focal lesions like tumour, infarct, gliosis, atrophy were revealed on CT-scan and magnetic resonance imaging (MRI) of the brain. Epilepsy was diagnosed when there was a history of two or more unprovoked seizures. These were classified with a simplified International League Against Epilepsy classification ${ }^{13-14}$ as generalized epilepsy, which included myoclonic seizures, infantile spasms, absence seizures, atonic seizures, generalized tonic-clonic seizures, generalized clonic seizures, or tonic seizures; partial epilepsy, which included simple or complex partial seizures, or secondarily generalized seizures and unclassifiable seizures, which were atypical or those in which the children were not sure whether they were focal or generalized in presentation ${ }^{13-16}$. By using standard clinical and investigation criterion epilepsy was further classified as 'symptomatic' if there was a clear antecedent history like significant head trauma, CNS infection and when a structural lesion was documented on neuroimaging and 'idiopathic' if there was no such evidence of a cerebral lesion. Patients with recurrent seizures, and with clinical or EEG evidence of focal onset but no evidence of causation were included under 'cryptogenic' localization related epilepsy. Epileptic disorders with mental retardation and frequent seizures which lacked the characteristic features of the defined syndromes were included under "other symptomatic generalized epilepsies not defined ${ }^{13-14}$. Partial seizures were further sub-classified chiefly on the basis of whether or not consciousness was impaired during the attack and whether or not progression to generalized convulsions occurred and these were simple partial seizures when consciousness was not impaired; complex partial seizures when consciousness was impaired; partial seizures like simple or complex evolving to secondarily generalized like tonic-clonic or tonic or clonic seizures. Complex partial seizures were further sub-classified chiefly on the basis of involvement of temporal lobe or not like complex partial seizures-Temporal (CPS-T) and complex partial seizures-extra-temporal (CPS-ET). Temporal lobe epilepsy was diagnosed if the history, seizure semiology, EEG and/or imaging showed evidence in favor of seizure originating over the temporal lobes. Rest of the CPS cases which did not fit to temporal lobe origin were considered to be originated over lobes other than temporal (CPS-ET). As the International League Against Epilepsy classification there was no special entity for 'Status Epilepticus' or 'Epilepsia Partialis Continua', the cases of EPC had been included in the partial seizure group. Statistical analysis was done by using SPSS version 21.0 for Windows. Assumptions of normality and homogeneity of variance were initially checked. Data were presented as median and ranges when distribution was asymmetric and mean and SD when distribution was normal. Categorical variables were expressed as proportions. The study protocol was approved by institutional ethical committee of Shaheed Sheikh Abu Naser Specialized Hospital, Khulna, Bangladesh

\section{Results}

The majority of patients attending outdoor clinic were $<30$ years age group (82.7\%) (Table 1). There was a male (77\%) and urban (53\%) predominance (Table 2,3).

Table 1: Age Distribution among the Study Population $(\mathrm{n}=115)$

\begin{tabular}{lcc}
\hline Age Group & Frequency & Percentage \\
\hline 0 to 9 Years & 34 & 29.6 \\
10 to 19 Years & 40 & 34.8 \\
20-29 Years & 21 & 18.3 \\
30-39 Years & 10 & 8.7 \\
40-49 Years & 05 & 4.3 \\
More Than 50 Years & 05 & 4.3 \\
\hline Total & $\mathbf{1 1 5}$ & $\mathbf{1 0 0 . 0}$ \\
\hline
\end{tabular}

Table 2: Gender Distribution among the Study Population $(\mathrm{n}=115)$

\begin{tabular}{lcc}
\hline Gender & Frequency & Percentage \\
\hline Male & 77 & 67.0 \\
Female & 38 & 33.0 \\
\hline Total & $\mathbf{1 1 5}$ & $\mathbf{1 0 0 . 0}$ \\
\hline
\end{tabular}

Urban patients were residing in cities and in municipal corporations. All patients other than from urban areas were called as rural patients 
Table 3: Residence of Patients

\begin{tabular}{lcc}
\hline Residence & Frequency & Percentage \\
\hline Rural & 54 & 47.0 \\
Urban & 61 & 53.0 \\
\hline Total & $\mathbf{1 1 5}$ & $\mathbf{1 0 0 . 0}$ \\
\hline
\end{tabular}

A large number of patients were student (40.9\%), followed by preschool child (12.2\%) and unemployed (10.4\%) (Table 4).

Table 4: Occupation of Patients $(n=115)$

\begin{tabular}{lcc}
\hline Occupation & Frequency & Percentage \\
\hline Preschool & 14 & 12.2 \\
Student & 47 & 40.9 \\
House wife & 8 & 7 \\
Business & 11 & 9.6 \\
Service & 8 & 7 \\
Unemployed & 12 & 10.4 \\
Others & 15 & 13 \\
\hline Total & $\mathbf{1 1 5}$ & $\mathbf{1 0 0 . 0}$ \\
\hline
\end{tabular}

The duration of epilepsy in most patients [67(58.2\%)] were less than 5 years (Table 5).

Table 5: Duration of epilepsy $(n=115)$

\begin{tabular}{lcc}
\hline Duration & Frequency & Percentage \\
\hline$<1$ yr & 28 & 24.3 \\
$1-5$ yrs & 39 & 33.9 \\
$5-10 y r s$ & 22 & 19.1 \\
$>10$ yrs & 26 & 22.6 \\
\hline Total & $\mathbf{1 1 5}$ & $\mathbf{1 0 0 . 0}$ \\
\hline
\end{tabular}

Among the 115 patients, 89 (77.4\%) had various forms of partial seizure, $19 \%$ (Table 6).

Table 5: Duration of epilepsy $(n=115)$

\begin{tabular}{lcc}
\hline Types of seizure & Frequency & Percentage \\
\hline Focal Motor Seizure & 1 & 0.9 \\
CPS-T & 53 & 46.1 \\
CPS-ET & 29 & 25.2 \\
Partial seizure with Sec gen & 4 & 3.5 \\
EPC & 2 & 1.7 \\
Symp. Gen Epilepsy & 9 & 7.9 \\
IGE & 6 & 5.2 \\
CAE & 2 & 1.7 \\
JME & 1 & 0.9 \\
Sturge weber syndrome & 1 & 0.9 \\
Gelastic seizure & 1 & 0.9 \\
Reflex seizure & 1 & 0.9 \\
Tuberous sclerosis complex & 1 & 0.9 \\
Pseudo seizure & 4 & 3.5 \\
\hline Total & $\mathbf{1 1 5}$ & $\mathbf{1 0 0 . 0}$ \\
\hline
\end{tabular}

CPS-T $=$ complex partial seizures-Temporal; CPS-ET $=$ complex partial seizures-extra-temporal; $\mathrm{EPC}=$ Epilepsia partialis continua; IGE $=$ Idiopathic generalized epilepsy; $\mathrm{CAE}=$ Childhood absence epilepsy; JME= Juvenile myoclonic epilepsy.
Among the partial seizure patients, 32\% patients are suffering from TLE and 59.5\% are suffering from Extra-TLE. $4.5 \%$ patients have PS with secondary generalization. In this study $9.7 \%$ patients have been suffering from symptomatic generalized epilepsy most of them are child and have history of perinatal asphyxia and head injury, 1 patient was found to have Tuberous sclerosis complex and 1 patient was found to have Sturge-Weber syndrome and 5.2\% patient have IGE (Table-7).

Table 7: Types of Partial Seizure and Their Distribution

\begin{tabular}{lcc}
\hline Types of Partial seizure & Frequency & Percentage \\
\hline Focal Motor Seizure & 1 & 1.1 \\
CPS-T & 53 & 59.6 \\
CPS-ET & 29 & 32.1 \\
Partial seizure with Sec gen & 4 & 4.5 \\
EPC & 2 & 2.2 \\
\hline Total & $\mathbf{8 9}$ & $\mathbf{1 0 0 . 0}$ \\
\hline
\end{tabular}

CPS-T $=$ complex partial seizures-Temporal; CPS-ET $=$ complex partial seizures-extra-temporal; EPC $=$ Epilepsia Partialis Continua

EEG was done in 76 patients and had found abnormalities in $37(48.7 \%)$ cases [Figure I]. Of them $18(23.7 \%)$ cases showed FED; $8(10.5 \%)$ cases showed GED; $10(13.2 \%)$ cases showed focal or generalized slowing and $39(51.0 \%)$ cases were normal (Table 8).

Table 8: EEG findings of the patients

\begin{tabular}{lcc}
\hline Findings & Frequency & Percentage \\
\hline Normal & 39 & 51.3 \\
FED & 18 & 23.7 \\
GED & 8 & 10.5 \\
Slowing & 10 & 13.2 \\
Others & 1 & 1.3 \\
\hline Total & $\mathbf{7 6}$ & $\mathbf{1 0 0 . 0}$ \\
\hline FED $=$ Focal Epileptiform & Discharge; & GED $=$ Generalized \\
Epileptiform Discharge & &
\end{tabular}

Among total 52 MRI, $61.5 \%$ showed various types of abnormal findings [Figure II] and among total $8 \mathrm{CT}$ Scan 50\% were abnormal (Table 9,10).

Table 9: CT scan findings of the patients

\begin{tabular}{lcc}
\hline Findings & Frequency & Percentage \\
\hline Normal & 4 & 50.0 \\
Abnormal & 4 & 50.0 \\
\hline Total & $\mathbf{7 6}$ & $\mathbf{1 0 0 . 0}$ \\
\hline
\end{tabular}




\section{Discussion}

Vast majority of the people in Bangladesh does have superstitious belief about Epilepsy. This belief usually is a strong barrier for total care of patients with epilepsy. Misunderstanding and negative attitude of the parents, family members and society towards epilepsy are very high. Thus, many patients with epilepsy are still neglected in diagnosis, treatment, education, rehabilitation and other social needs. The epilepsy patients are often reluctant to seek advice from physicians. Rather they believe epilepsy has no cure and they seek advice from indigenous medicine practitioner 'Kabiraj', snake charmer 'Ojha' and spiritual healers.

Table 10: MRI findings of the Patients

\begin{tabular}{lcc}
\hline Findings & Frequency & Percentage \\
\hline Normal & 20 & 38.5 \\
Abnormal & 32 & 61.5 \\
\hline Total & $\mathbf{5 2}$ & $\mathbf{1 0 0 . 0}$ \\
\hline
\end{tabular}

A report of 130 patients from the epilepsy clinic of BSMMU, a public medical university and post-graduate medical center, showed that close to $70 \%$ of patients visited indigenous medicine practitioners, exorcists, spiritualists prior to consulting the clinic, only $29 \%$ perceived epilepsy as a disease, $50 \%$ dropped out from school $58 \%$ of whom due to epilepsy and $52 \%$ of patients had to change job because of epilepsy. Appropriate antiepileptic drugs are sometimes very costly or unavailable in Bangladesh. The BSMMU study showed $23 \%$ of patients found it difficult to continue treatment due to financial problem. Financial factor is likely to partly account for the treatment gap ${ }^{6}$.

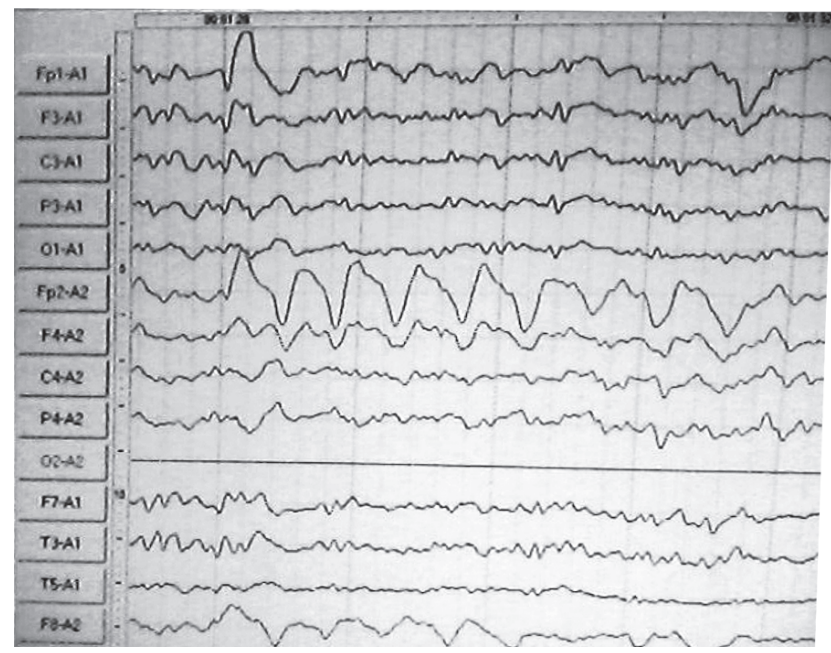

Figure I: EEG showing Frontal Intermittent Rhythmic Delta Activity (FIRDA) where CT-scan Showed Infarction in Right Frontal Lobe
In this study there is slight urban predominance (53\%). Two studies (in Pakistan and in India) indicated that the prevalence was higher in rural areas than in urban areas. A meta-analysis of published and non-published community-based studies in India showed a higher prevalence rate of epilepsy of 5.5 per $1000(95 \%$ CI 4.0-6.9) in rural areas than that of 5.1 per 1000 (3.5-6.7) in urban areas18. Again in some studies it is also found that urban men and women had a higher prevalence of epilepsy compared with rural ones, however the difference was not statistically significant ${ }^{17}$.

Based on the findings of this study several issues demand attraction. The most common age of presentation of epilepsy was $<30$ years $(82.7 \%)$. In a study, Sridharan and Murthy ${ }^{17}$ showed similar findings 'age-specific prevalence rates were higher in the younger age group, with the onset of epilepsy reported mostly in the first three decades of the sample population's lives'. Except for Shanghai in China, most of the Asian countries have younger epileptic patients. The probable reason for the missing peak in the older age group in many Asian countries is due to the fact that the population in general is younger ${ }^{18}$.

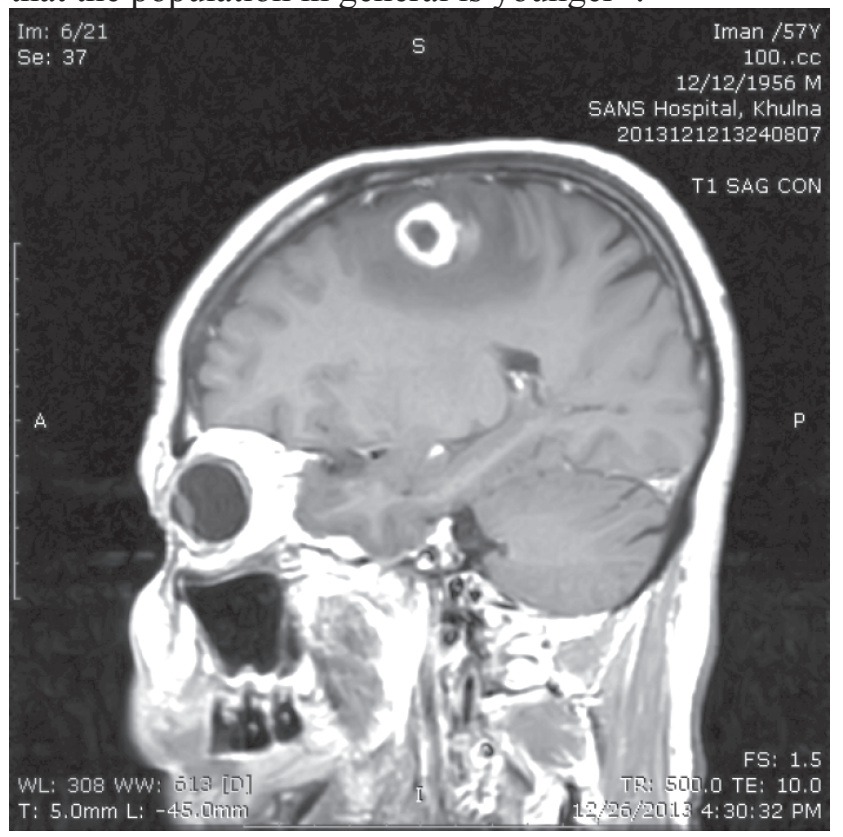

Figure II: MRI showing Tuberculoma

Here a male predominant (77.0\%) picture is seen. Epilepsy is slightly more common in men than in women but the sex-specific prevalence is not, in general, significantly different ${ }^{18}$. Reports are similar in other Asian countries ${ }^{19}$. Although our country has almost same socio-economic condition like the 
surrounding countries (India and Pakistan) here women are more home bound and neglected. Regarding epilepsy various social stigma remains. So people try to hide the illness of their daughters and sisters. As males are more exposed to outer world, their diseases come to the attention easily and people bring them to doctors. Also, as they are the earning members, their illness gets more importance for the uninterrupted continuation of earning source.

The students $(41.0 \%)$ came to epilepsy clinic as they are the knowledgeable group of society who have an easy access to a tertiary health care system. They draw attention through their teachers and fellows and also the parents are more concerned with the illness of their kids. This is a good sign that a major portion $(58 \%)$ of patients presented to the clinic within 5 years of their illness which may be due to good referral system of the hospitals and doctors. Even then a significant portion of the population is attending late. This has to be addressed by our health professionals and policy makers.

Regarding distribution of seizures $77.0 \%$ patients are suffering from various forms of partial seizure. Studies from India also recorded a high frequency of partial epilepsies which were $62.9 \%{ }^{20}$ and $57.0 \%{ }^{21}$ cases. Data in other developing ${ }^{22-25}$ and developed countries shows same finding ${ }^{27}$. Earlier study of Bangladesh also showed a high frequency of partial epilepsies which was $54.0 \%{ }^{26}$.

In this study extra-temporal lobe epilepsy comprises $32.0 \%$ which appears to be relatively more than other's finding. This may be due to the misinterpretation of people of the psychic and non-convulsive pattern of TLE, for which they usually seek help from traditional and spiritual healers or they go to the psychiatrist at best. The extra-temporal lobe epilepsy patients usually present with convulsion and draw attention of the attendants and physicians early. But the major portion of non-convulsive seizure patients are suffering from TLE which are difficult to diagnose, needs high index of suspicion and advanced training on epilepsy. Many patients showed lesions (gliosis, atrophy in MRI) in occipital and posterior head region which indicates birth injury, asphyxia and trauma which may be the reason for increased number of Ex-TLE in this area.

Although few patients were ultimately diagnosed as having pseudo-seizure, this issue should be taken into account as all of them were getting anti-epileptic drugs along with all the restrictions and burden of epilepsy. EEG findings were positive in almost $49.0 \%$ cases. This also matches with the finding by other authors ${ }^{28}$.
There are some limitations in the study. I could not apply the recent ILAE proposal for classification of epilepsy syndrome. The study from hospital records may not completely represent the scenario in the community. Finally, also due to the retrospective nature, some aspects like the attitude of patients and attendants to epilepsy could not be evaluated.

\section{Conclusion}

This study highlights some facts. LRE are more common in Bangladesh. Most of them are symptomatic and cryptogenic. Therefore, facilities should be provided to identify the etiology. The high number of Ex-TLE needs further evaluation in big sample in our country. This demands further study in big sample in this area.

\section{References}

1. Meinardi H, Scott RA, Reis R, Sander JW. The treatment gap in epilepsy: the current situation and ways forward. Epilepsia 2001;42:136-4

2. Ngugi AK, Bottemley C, Kleinschmidt I., et al. Estimation of the burden of active and life-time epilepsy: a meta-analytic approach. Epilepsia 2010; 51: 883-90

3. Bharucha NE. Epidemiology of Epilepsy in India. Epilepsia 2003;44(Suppl. 1):9-11

4. Neligan A, Sander JW. The incidence and prevalence of epilepsy. London: UCL Institute of Neurology. 2009

5. Banu S, Khan N, Hossain M, Jahan A, Parveen M, Rahman N, et al. Profile of childhood epilepsy in Bangladesh. Dev Med Child Neurol. 2003;45:477-82

6. Mannan MA. Epilepsy in Bangladesh. Neurology Asia 2004; 9 (Supplement 1): 18

7. WHO report on Epilepsy in South East Asia. Some facts and figures in Epilepsy. Available from:www.searo.who.int/LinkFiles/Information and Documents facts

8. Bangladesh Bureau of Statistics(BBS), Report 2011

9. Commission on Epidemiology and Prognosis, International League Against Epilepsy. Guidelines for epidemiologic studies on epilepsy. Epilepsia 1993; 34:592-6

10. Rajasekhar V, Haran RP, Prakash GS, Chandy MJ. Differentiating solitary small cysticercus granulomas and tuberculomas in patients with epilepsy: clinical and computerized tomographic criteria. J Neurosurg 1993; 78:402-7

11. Sundaram C, Murthy VS, Dinakar I, Ratnakar KS. CT and histological correlation of intracranial tuberculomas. Neurol India 1989; 37(suppl): 188

12. Rajasekhar V. Etiology and management of single small CT lesions in patients with seizure: understanding a controversy. Actu Neurol Scand 1991; 84:465-70

13. ILAE. Commission on Classification and Terminology of International League Against Epilepsy: proposal for revised classification of epilepsies and epileptic syndrome. Epilepsia 1989;30: 389-99

14. ILAE. Commission on Epidemiology and Prognosis of the ILAE. Guidelines for epidemiologic studies on epilepsy. Epilepsia 1993;34: 592-6

15. Avanzini G, Franceschetti S, Binelli S, Panzica F, Granata T, Canger R, et al. ILAE classification of epilepsies. Epilepsia. 
1996;37(11):1051-9

16. Beilmann A, Talvik T. Is the ILAE classification of epileptic syndromes applicable for children in Estonia? Eur J Paediatr Neurol 199;3: 265-2

17. Sridharan R, Murthy BN; Prevalence and pattern of epilepsy in India. Epilepsia 1999;40(5):631-6

18. Mac T, Tran D, Quet F, Odermatt P, Preux P, Tan C. Epidemiology, aetiology and clinical management of epilepsy in Asia: a systematic review. Lancet Neurol 2007; 6:533-43

19. Fong G, Mak W, Cheng T, Chan K, Fong J, Ho S. A prevalence study of epilepsy in Hong Kong. Hong Kong Med J 2003;9:252-7 20. Murthy JM, Yangala R, Srinivas M. The Syndromic Classification of the International League against Epilepsy: A Hospital Based Study from South India. Epilepsia. 1998;39(1):48-54

21. Mani KS, Rangan G. Epilepsy in the Third World: Asian aspects. In: Dam M, Gram L, eds. Comprehensive epileptology. New York: Raven Press, 1990:781-93
22. Matuja WBP. Aetiological factors in Tanzanian epileptics. East Afr Med J 1989; 66:343-8

23. Danesi MA. Classification of the epilepsies: an investigation of 945 patients in a developing country. Epilepsia 1985;26:13 1-6

24. Senanayake N. Classification of epileptic seizures: a hospitalbased study of 1,250 patients in a developing country. Epilepsia 1993;34:8 12-8

25. Tan CT, Lim SH. Epilepsy in South East Asia. Neurol J Southeast Asia 1997;2:11-15

26. Khan SU. Characteristics of epilepsy patients at a tertiary care hospital in Bangladesh. Research 2014;1:74

27. Zarrelli MM, Beghi E, Rocca WA, Hauser WA. Incidence of epileptic syndromes in Rochester, Minnesota: 1980-1984. Epilepsia 1999;40(12):1708-14

28. Smith SJM. EEG in the diagnosis, classification, and management of patients with epilepsy, J Neurol Neurosurg Psychiatry 2005;76:ii2-ii7 